Data are collected in real time, and all partner notification outcomes are recorded. The platform is extendable to facilitate centralised partner notification within sexual health clinical networks across many different heath care providers.

\section{P111 A USER CENTRED APPROACH TO THE DESIGN OF POINT- OF-CARE AND SELF-TEST MOBILE PHONE DIAGNOSTICS FOR SEXUALLY TRANSMITTED INFECTIONS (STIS)}

\author{
doi:10.1136/sextrans-2012-050601c.111
}

${ }^{1} \mathrm{G}$ Voula, ${ }^{*}{ }^{1} \mathrm{~W}$ Balachandran, ${ }^{2} \mathrm{C}$ Lowndes, ${ }^{2} \mathrm{R}$ Howell-Jones, ${ }^{3} \mathrm{C}$ R Aicken, ${ }^{3} \mathrm{C} H$ Mercer, ${ }^{4} \mathrm{~L}$ Sutcliffe, ${ }^{3} \mathrm{P}$ Sonnenberg, ${ }^{5} \mathrm{M}$ Jackson, ${ }^{4} \mathrm{C}$ S Estcourt, ${ }^{6} \mathrm{~S}$ T Sadiq, ${ }^{1} \mathrm{~K}$ Hone. ${ }^{1}$ Brunel University, Middlesex, UK; ${ }^{2}$ Health Protection Agency; ${ }^{3}$ University College London, London, UK; ${ }^{4}$ Queen Mary University of London; ${ }^{5}$ xscient; ${ }^{6}$ St Georges University of London

Background Control of STIs in vulnerable populations is difficult because of inadequate access to prompt diagnosis and treatment and may be enhanced by better community based STI surveillance. The high rates of mobile and smart phone use in these populations and the development of novel personalised diagnostic technologies which theoretically enable highly accurate self-testing diagnostics for STIs that can be electronically linked to clinical care pathways and STI data capture, may help address these challenges. A major challenge for these systems is the development of clinically relevant and acceptable Human Technology Interfaces (HTIs) for Mobile-Web Applications. Objectives To describe a methodological approach to develop a mobile phone/web-based management system that can link rapid self-test diagnostics to clinical care pathways and community based surveillance.

Methods We adopt a user centred approach to the development of a HTI for self-managing STI diagnosis with initial exploratory pilot studies to gather functional, user and clinical requirements of the HTI. Iterative development of functional prototypes exploring design possibilities and technology features is followed by the formative evaluation of interface design alternatives through qualitative and controlled usability studies with target users and existing surveillance capture mechanisms.

Results A user centred, multi-disciplinary approach allows for successful capture and integration of social science methods to inform functional requirements for an effective and user acceptable mobile and web-based self-managing STI diagnostics and surveillance system.

Discussion Future testing in simple and complex, community based studies are needed to primarily assess safety and then effectiveness for improving access to care, enhanced partner notification and development of community based capture of surveillance data.

\section{P112 TEXTING OR TALKING? WHICH WORKS BETTER TO REDUCE HAZARDOUS DRINKING BY SEXUAL HEALTH CLINIC ATTENDEES?}

doi:10.1136/sextrans-2012-050601c.112

\section{S Baguley.* NHS Grampian}

Background Hazardous drinking is associated with an increased risk of catching an STI, getting pregnant unintentionally, being sexually assaulted and sexually assaulting someone else. An Alcohol Brief Intervention ( $\mathrm{ABI}$ ), a type of motivational interview, has been shown to reduce hazardous drinking in men over the age of 25 . There is an paucity of evidence of the benefit in younger people or in women. Texting has been shown to be useful in supporting smoking cessation, weight reduction and diabetes control. There have been no studies of its potential value in reducing hazardous drinking or its consequences.
Aim To compare the effect of texting vs an ABI on hazardous drinking and associated sexual illhealth amongst attendees at an urban sexual health clinic.

Methods Potential subjects are being recruited from a city sexual health clinic. Initial screening is done using the Alcohol Use Disorder Identification Test - a self-completed questionnaire marked out of 40. Consenting subjects are then randomised to texting (12 texts over 4 months), an ABI or a leaflet and then followed up by phone at 4 months. At follow-up, subjects are rescreened for hazardous drinking and asked about incident STIs, regretted sex, sexual assault, use of emergency contraception and for their evaluation of the intervention method.

Results To end of January 2012, 6279 attendees have been screened of whom 2554 were hazardous drinkers and 799 have consented to take part in the study. (The target sample size is 1090). By the time of the conference, all subjects will have been recruited and most results will be available.

Conclusion This is the first RCT of texting for hazardous drinking and should inform national policy in this area.

\section{P113 PRIMARY HEALTH PROVIDERS' ADHERENCE TO NATIONAL CHLAMYDIA GUIDELINES}

doi:10.1136/sextrans-2012-050601c.113

\section{${ }^{1} \mathrm{~J}$ M Morgan, ${ }^{* 2} \mathrm{~L}$ A Mills, ${ }^{1} \mathrm{~A}$ J Bell. ${ }^{1}$ Waikato Hospital; ${ }^{2}$ University of Otago}

Aim To re-audit district-wide management of genital chlamydia infection following implementation of $\mathrm{NZ}$ national chlamydia guidelines.

Methods All healthcare settings in a single NZ health district that diagnosed 20 or more cases of chlamydia during September 2010-2011 were invited to participate and were asked to complete an audit proforma for 20 consecutive cases. Sexual Health clinic cases were excluded. Collated results were compared to findings of a similar audit of 394 cases diagnosed during 2008, which was undertaken before guideline implementation in 2009.

Results 19 of 22 eligible sites participated, providing data for 424 cases; of these, 302 (71.2\%) were managed in General Practice, with the remainder from a range of settings including Family Planning, student health services, and A\&E. 405 of 424 (95.5\%) cases in 2010-2011 had documented treatment compared to 354 of 394 $(89.8 \%)$ in 2008. Notifying current sexual partners was noted in 314 cases $(74 \%)$, compared to $58.1 \%$ in 2008.126 of 424 (29.7\%) of cases were noted to have informed at least $50 \%$ of their sexual partners compared to $17.8 \%$ in 2008 .

Conclusion Improved case management and partner notification may reflect better documentation than in the initial audit but more appropriate choice of diagnostic sampling, more testing for other STIs and more appropriate dosage and duration of prescribed antibiotics supports that primary care chlamydia case management in this district has improved following local implementation of national guidelines. More work is needed to enhance documented partner management outcomes.

\section{Improving clinical practice and service delivery}

\begin{tabular}{l|l}
\hline P114 IS THERE A ROLE FOR PRIMARY CARE CLINICIANS IN \\
PROVIDING SHARED CARE IN HIV TREATMENT? A \\
SYSTEMATIC LITERATURE REVIEW
\end{tabular}

doi:10.1136/sextrans-2012-050601c.114

${ }^{1}$ W Wong, ${ }^{1} \mathrm{C}$ W Luk, ${ }^{2} \mathrm{M}$ R Kidd. ${ }^{1}$ The University of Hong Kong; ${ }^{2}$ Faculty of Health Sciences, Flinders University

Background Despite HIV being increasingly considered as a chronic illness, there is as yet no consensus about how primary care should 